HOFFMANN, O. (2002) "De paysans à indiens, les représentations de la paysannerie latino-américaine, de 1970 à 2000", Caravelle No79, p9-19.

\title{
De paysans à indiens, les représentations de la paysannerie latino-américaine, de 1970 à 2000
}

\author{
Odile Hoffmann \\ Directrice de recherche à l'IRD (Institut de Recherche pour le Développement, Paris) \\ mail: hoffmann@bondy.ird.fr
}

Depuis les années 1970, les conditions de vie en milieu rural ont été bouleversées dans de nombreux pays d'Amérique latine. Les paysans se sont de plus en plus intégrés aux logiques internationales d'échange, de production et de régulation. Ils sont nombreux, pour autant, à "rester sur le carreau" et à subir une globalisation porteuse d'exclusion et de pauvreté. Sans polémiquer sur la définition de "paysan" ni pour autant faire l'impasse sur une nécessaire définition de l'objet de la réflexion, je retiendrai, pour caractériser la paysannerie, les idées d'autonomie, de monde rural et de "rapport à la terre". Elles sont certes éminemment relatives puisqu'elles ne prennent sens que dans des sociétés nationales diversifiées, et dans des contextes spatio-temporels précis. Mais elles constituent, plus que d'autres (la production agricole, l'autosubsistance, les structures socio-familiales), les constantes qui, sous des appellations diverses, reviennent dans les approches scientifiques des "paysanneries".

Comment, en trente ans, ont évolué les regards universitaires sur les paysans latinoaméricains et leurs familles ? En quoi les évolutions de la pensée scientifique ont-elles permis de mieux comprendre les sociétés paysannes, et parfois de contribuer à leur transformation? Ces quelques lignes se voudraient une invitation à une réflexion sur les modes de pensée dans leurs rapports au politique d'une part, aux grandes transformations économiques d'autre part. Elles s'appuient sur des travaux menés au Mexique et en Colombie depuis le début des années 1980 et ne prétendent donc pas à l'exhaustivité, mais se veulent plutôt des pistes de réflexion sur le travail de chercheur et sa pertinence pour comprendre les changements passés et actuels des paysanneries latinoaméricaines. Je distingue trois phases principales dans l'évolution des théories, associées aux grandes étapes de la globalisation latino-américaine.

Les paysans face au capitalisme

Dans les années 1970-1980, le débat scientifique tourne autour de la définition des paysans, et de leur place dans des systèmes capitalistes marqués notamment par une urbanisation rapide. A cette époque, le monde rural alimente le développement industriel et urbain, par les migrations principalement, mais aussi par la ponction systématique des ressources "paysannes" : maintien de bas prix aux produits alimentaires de base, peu d'investissement social ni d'infrastructures en milieu rural. On se pose alors la question de la "prolétarisation" du paysan et de son devenir dans des sociétés "modernes". Marqué par les approches marxistes, le débat se nourrit aussi des 
mobilisations sociales paysannes vigoureuses, principalement autour de l'accès inégal à la terre. C'est l'époque des réformes agraires, qu'elles soient d'inspiration "révolutionnaire" -le droit à la terre- ou portées par l'Alliance pour le Progrès et les gouvernements conservateurs (pour la Colombie, cf. Gilhodes 1974).

L'argumentation reprend l'équation paysans $=$ terre $=$ autosubsistance et petite production, opposée aux " agriculteurs " modernes (classification CEPAL), dans des pays historiquement fondés sur l'antagonisme minifundio-latifundio comme on disait alors. Il s'agit, pour de nombreux chercheurs travaillant sur et avec les paysans, de légitimer un certain mode vie et de réclamer les besoins correspondants, dans un combat pour plus de justice et d'égalité sociale. Politiquement "de gauche", les courants majoritaires échappent rarement à une certaine idéalisation du paysan, sujet historique à défendre contre les grands propriétaires et les capitalistes.

Cette période a donné lieu à des débats intellectuels intenses ainsi qu'à une production scientifique abondante fondée sur les théories anthropologiques et sociologiques les plus novatrices du moment et associée à de réels travaux de terrain, d'une part, à des engagements personnels et politiques des chercheurs, d'autre part (Warman 1972). Au Mexique par exemple, les débats furent très vifs (cf. la polémique entre les anthropologues qualifiés de "paysannistes" retardataires et leurs détracteurs à leur tour accusés de "réactionnaires" modernisants) et les positions eurent tendance à se durcir et à fonctionner à base d'images d'Epinal, avec un danger croissant de caricaturer les réalités et de tomber dans des simplifications outrancières.

Cette polémique scientifique s'est essouflée à la fin des années 1980. D'une part, l'excès de théorisation qui tournait à l'absurde, ainsi que les impasses politiques et les échecs répétés des mobilisations paysannes, ont eu raison de cet "enthousiasme" universitaire pour le monde rural et paysan. D'autre part - et surtout ?- les études rurales "ne payaient plus". Sur le plan académique, les efforts théoriques portaient sur la ville et les processus naissants de globalisation. L'avenir des pays latino-américains était urbain : les taux d'urbanisation grimpaient jusqu'à 50 et même $70 \%$ (en Colombie par exemple). Les défis politiques et pratiques étaient immenses, la "demande sociale" de recherche et plus précisément celle des gouvernements et des agences internationales se focalisa sur les villes et/ou les phénomènes économiques et démographiques associés.

Ce retournement de tendances, c'est-à-dire la désaffection des chercheurs pour les mondes paysans et ruraux, fut assez radical pour provoquer un vide, une véritable lacune : en 1994 au Mexique, immédiatement après le soulèvement des Chiapas, les politiques cherchaient désespérément des "spécialistes" pour "expliquer" une situation que personne n'avait vu venir sauf exceptions, ou déclarations a posteriori.

La "nouvelle ruralité" et la modernisation des campagnes

Dans la phase suivante, qui couvre en gros la décennie des années 1990, les chercheurs renoncent aux théorisations englobantes et s'attachent à démontrer le caractère moderne et complexe des sociétés rurales et paysannes. En réaction aux positions tranchées de la période précédente, ils insistent sur les capacités d'adaptation des paysans, les interprétant parfois comme des formes de "résistance" politique et culturelle. Non pas une résistance d'arrière garde à la modernité, mais bien au contraire "le pouvoir des sans-pouvoir". Il s'agit de redonner aux paysans une parole et 
une initiative que les théories antérieures avaient eu tendance à sous-évaluer, focalisées comme elles l'étaient sur les processus de domination et d'exploitation. On cherche alors à sortir des schémas réducteurs gros/petits, forts/faibles, afin de montrer le dynamisme des sociétés locales, leurs transformations permanentes et leur potentiel de participation politique, dans un contexte de démocratisation dans tout le sous-continent. Moins encadrée qu'auparavant par des théories confirmées, la recherche n'en conserve pas moins ses ambitions conceptuelles. Elle déplace ses centres d'intérêt et fabrique même certains "gros mots" : la rurbanisation, la glocalisation, la "nouvelle ruralité", qui sont autant d'efforts pour exprimer la complexité de mondes pris, peutêtre plus que d'autres, entre le local et le global, la tradition et la modernité. Les paysans, pour la plupart, restent "attachés à la terre" tout en diversifiant considérablement leurs réseaux et leurs ressources : notamment avec les migrations, mais aussi le travail temporaire ou la multi-activité familiale. Le terme de "paysannerie" prête à confusion car trop connoté par les analyses antérieures ; on lui préfère alors celui de société locale, ou rurale, pour bien signifier le poids des interdépendances entre les acteurs et les sphères de niveaux variés.

Comme dans la période précédente, on peut relier ces tendances intellectuelles aux transformations sociales, économiques et politiques du moment. Les mobilisations paysannes ont faibli, la lutte pour la terre ne trouve plus d'écho politique et les "paysans" -car ils continuent bien d'exister, eux!- s'organisent autour de revendications liées à l'accès aux services et/ou la commercialisation de leurs produits pour sortir de la dépendance. Au Mexique par exemple, c'est l'époque de la lutte pour le contrôle des instances municipales, de l'organisation des habitants pour réclamer écoles ou centres de santé, ou encore des producteurs (de café notamment) autour des coopératives de transformation et commercialisation. L'autonomie locale et le refus de la domination restent bien au coeur des logiques paysannes, mais ils sont désormais exprimés sur d'autres fronts, moins corporatistes que précédemment. Les recherches universitaires traduisent et accompagnent ces évolutions. Les "paysans" sont compris d'abord comme des "habitants" des espaces ruraux qu'ils maitrisent en partie, et comme des acteurs sociaux à la recherche d'autonomie et de participation à la société nationale. En cela aussi, la recherche est en phase avec les théories sociales du moment qui parlent de société civile et de démocratie participative. Le paysan est un "sujet" politique, un acteur social (Touraine 1988).

Le rapport à la terre, encore et toujours... mais différemment

La troisième phase démarre avec l'application des politiques néoliberales. Contrairement à ce qu'avaient cru les modernisateurs des années 1970, la question du rapport à la terre reste le défi majeur des paysanneries et mondes ruraux contemporains. Evacuée pendant un moment, elle revient sur la scène à la fin des années 1990, mais sous des perspectives renouvelées dont deux principales : économique avec une réflexion sur la place de la terre dans un système de marché, anthropologique et géographique sous l'angle du territoire, lié à la question des identités. Toutes deux se situent dans la droite ligne des débats internationaux qui portent sur la libéralisation des marchés d'une part, sur le respect des "minorités" et le multiculturalisme d'autre part.

L'approche économique tend à réduire la question foncière à la mise en place de dispositifs qui favorisent la circulation de la terre, conçue comme une marchandise, condition nécessaire pour attirer les investissements fonciers et agricoles. Les efforts portent dès lors sur la normalisation et la régularisation juridique des modes de possession et d'appropriation de la terre. En effet, ceux-ci 
étaient le fruit d'évolutions historiques (notamment des lois de réforme agraire) qui avaient donné naissance à de multiples configurations légales : propriété, communauté, colonie agricole, ejido, mais aussi possession de facto sans titre juridique, réserve indienne, et toutes les formes de métayage, prêt et location de longue durée qui, jusqu'à une date récente, régulaient socialement l'accès légitime des paysans à la terre. Ces figures juridiques, élaborées en leurs temps pour tenir compte des particularités économiques ou ethniques des paysans et parfois pour les préserver des appétits fonciers des grands propriétaires, restreignent en général les droits de circulation (interdits de vente, mise en gage ou location). Fondés sur des critères politiques des périodes précédentes (intervention de l'Etat), ils sont désormais des obstacles à la libéralisation généralisée des échanges. Pour les théoriciens comme pour les dirigeants politiques nationaux, il faut alors "clarifier les droits de propriété", condition présentée comme indispensable à la sécurisation foncière légale et à une plus grande circulation marchande des terres.

Aujourd'hui la plupart des pays latino-américains mettent en place des mécanismes tendant à favoriser un marché de terres avec l'appui d'organismes internationaux (notamment la Banque Mondiale et la FAO). Les conséquences ne sont pas encore évidentes. Certains craignent une dynamique rapide de vente de terres paysannes au profit des agriculteurs-éleveurs et des entreprises agro-industrielles, ce qui menacerait à court terme les bases mêmes de la reproduction sociale et économique des sociétés rurales paysannes. Avéré localement (au Mexique dans les zones d'horticulture de haut rendement, en Colombie dans les régions de plantations de palmiers à huile par exemple), ce processus ne s'est pourtant pas généralisé, tant il est vrai que la rentabilité de l'agriculture a chuté ces dernières décennies au point de ne plus représenter un champ d'investissement pouvant intéresser les firmes capitalistes. Les prix des produits agricoles ne cessent de baisser et, pour les entrepreneurs agricoles, la sécurisation foncière ne passe plus nécessairement par la propriété : les contrats légaux de métayage et de location sont souvent bien plus profitables et flexibles, leur laissant la possibilité de se délocaliser lorsque les conditions de production se dégradent (pour des motifs agro-écologiques d'épuisement des sols par exemple). La libéralisation des terres, si elle n'a pas abouti à des processus massifs de concentration foncière, n'en a pas moins accru la dépendance des petits agriculteurs vis-à-vis des marchés et des grands entrepreneurs agricoles.

A ces raisons d'ordre strictement économique qui expliquent le maintien d'une petite propriété paysanne, s'ajoutent des raisons d'ordre politique, parfois interprétées comme ressortant du registre symbolique ou culturel. Contrairement aux calculs économiques qui postulent la marchandisation de la terre, celle-ci reste associée à d'autres dimensions non marchandes. En effet, l'accès à la terre conditionne et légitime la position des paysans dans les sociétés locales. Que ce soit dans les équilibres entre les groupes de pouvoir locaux, ou au sein même des unités familiales, la possession socialement légitime -qu'elle qu'en soit la forme juridique- d'une portion de terre ouvre l'accès aux réseaux sociaux et politiques qui structurent les sociétés rurales. Les paysans mexicains qui appartenaient au "secteur social" ne s'y sont pas trompés : au moment de la mise en place des politiques de privatisation de la terre, ils ont opté massivement pour la régularisation foncière mais n'ont pas réalisé la phase ultime du programme, PROCEDE, qui aurait abouti à la privatisation individuelle. Ils conservent ainsi le statut ejidal qui leur assure une "place à part" dans la Nation, collective et politique, et l'accès correspondant aux dispositifs de pouvoir local et régional. Ce n'est pas tant "l'attachement à la terre" que la compréhension intime de ses fonctionalités multiples, qui explique le maintien de stratégies foncières "paysannes". 
Quand le paysan s'efface devant l'indien...

La dernière approche que je veux mentionner ici, qui se développe dans le même temps que la précédente, concerne le "renouveau des identités" en milieu rural. Ili affecte l'ensemble du souscontinent depuis les années 1970-80, mais acquiert un poids spécifique depuis sa traduction en termes constitutionnels dans les années 1990, dans plusieurs pays qui se déclarent désormais multiculturels et pluri-ethniques. Tout se passe comme si, pour les paysans, les revendications agraires et politiques ayant en grande partie échoué, la demande de participation citoyenne se soit orientée vers ce nouveau champ de légitimité. Au Mexique, les politiques de reconnaissance ethnique ont une longue histoire institutionnelle (notamment à travers l'INI, Institut National Indigéniste) et se réactivent sous le gouvernement de Carlos Salinas de Gortari (1988-1994) avec des mesures spécifiques destinées aux groupes indiens (protection du patrimoine culturel, développement des infrastructures en régions indiennes). Le soulèvement néozapatiste de 1994, en obligeant la société toute entière à ouvrir les yeux sur les réalités paysannes indiennes, va brusquement accélerer et amplifier la mise en place de mesures spécifiques, dans tout le pays et pas seulement au Chiapas. Si l'on veut bien exclure les polémiques autour de la figure de Marcos ${ }^{1}$, il faut reconnaitre que l'insurrection a suscité des débats de fond qui agitent le pays et le monde universitaire et politique comme ils ne l'avaient pas fait depuis les années 1970. La place des indiens dans la Nation à l'heure de la globalisation, le rapport des paysans et des indiens, la démocratie et les structures corporatistes de pouvoir local et régional, sont autant de thèmes qui interrogent les théories actuelles, bien que fondés sur des exigences qui n'ont rien de bien nouveau : justice sociale et dignité.

La "question ethnique" revient sur la scène politique des pays qui pensaient l'avoir évacuée au moyen de politiques sectorielles (assistance à la pauvreté). Les organisations internationales ont un rôle fondamental dans ce renouveau de la mobilisation : l'Organisation Internationale du Travail, OIT, dès 1989 avec son décret 169 qui reconnait les droits des peuples indigènes et tribaux, signé par la plupart des pays, et plus tard la Banque Mondiale qui emboite le pas aux ONG mobilisées autour du respect des droits humains (notamment en Amazonie). Dans les régions rurales des Amériques, les organisations indépendantes locales peuvent alors s'appuyer sur cet "arsenal" pour visibiliser leurs revendications en les réinterprétant sous l'angle ethnique. Il s'agit tout à la fois d'une "invention", au sens d'une construction politique nouvelle, et d'une récupération de demandes anciennes qui n'avaient jamais été écoutées (justice, égalité, participation). La "re-indianisation" de plusieurs pays d'Amérique latine doit donc se comprendre comme une exigence renouvelée de participation citoyenne à la Nation, empruntant des registres qui avaient été dé-légitimés dans le passé et qui retrouvent aujourd'hui droit de cité. Dans ce contexte, le "territoire ethnique" devient la preuve et la condition de l'autonomie socio-politique (au sens anthropologique et non pas juridico-légal du terme), et les discours sur "le rapport ancestral à la Terre", certes fondés culturellement et historiquement, deviennent surtout des instruments de négociation entre les groupes indiens (ou noirs, ou caboclos, etc..) organisés et les gouvernements.

\footnotetext{
${ }^{1}$ Je ne fais ici allusion qu'à la dimension nationale et internationale du débat. L'insurrection a provoqué des bouleversements locaux et régionaux d'une complexité telle -sur les plans économique, social, politique et culturelque seules des analyses détaillées et bien documentées peuvent en rendre compte.
} 
La recherche accompagne ces bouleversements, s'appuyant sur les théories qui précisent les mécanismes de construction et d'invention des identités, depuis le niveau local jusqu'au national (les communautés imaginées, l'invention de la tradition). Paradoxalement, au moment où "l'identité ethnique" devient une catégorie monneyable sur le plan politique, elle est unanimement relativisée par les scientifiques qui en soulignent les aspects construits et instrumentalisés : “on ne nait pas indien (ou noir), on le devient". Pourtant, la dimension ethnique n'est pas seulement une nouvelle ressource dont usent les paysans et leurs organisations pour se positionner dans les sociétés locales, régionales et nationales ; elle est aussi, désormais, reconnue comme une "valeur" légitime qui organise la compréhension du monde et oriente les pratiques sociales, culturelles, politiques et maintenant économiques de nombreux acteurs.

En Colombie par exemple, la reconnaissance des communautés noires et de leurs droits fonciers sur les territoires qu'elles occupent ancestralement (Constitution de 1991 et Loi 70 de 1993) modifie en profondeur l'image qu'ont d'eux-mêmes les habitants concernés. De paysans pauvres oubliés de la société nationale, ils deviennent sujets politiques en tant que "communautés noires" reconnues par l'Etat et les institutions, mais aussi par les fonctionnaires, les hommes et femmes politiques, les autres habitants. La dimension instrumentale de l'ethnicité, certes réelle, s'accompagne d'une véritable recomposition des sociétés nationales qui doivent intègrer la différence ethnique dans leurs conceptions d'elles-mêmes et de leurs devenirs. A partir de revendications territoriales, les organisations noires se projettent sur la scène publique et luttent contre la discrimination et pour de meilleures conditions d'insertion dans la société nationale. Le monde rural, à l'origine de la mobilisation ethnique, occupe les premiers rangs dans ce que l'on interpréter comme une recomposition idéologique de l'idée de Nation.

Comme ailleurs, ce changement de paradigme peut faire peur : les politiques multiculturalistes peuvent entretenir ou même engendrer des dynamiques communautaristes et particularistes qui menaceraient l'idée de communauté nationale. Pourtant, du point de vue des acteurs concernés, il s'agit précisément du contraire: de transformer des Nations éclatées, de les "réinventer" sur un mode de démocratie participative et citoyenne jusqu'ici absente.

On note aisément les contradictions entre les deux derniers courants mentionnés : entre une libéralisation des marchés qui individualise le rapport à la terre, et un multiculturalisme qui au contraire le collectivise sous forme de "territoire ethnique". Paradoxalement, les deux sont légitimes aux yeux des dirigeants nationaux et des agences internationales; les deux conditionnent les crédits et les programmes "d'aide au développement" ; les deux contribuent aux transformations actuelles de l'Amérique latine. Les paysans, au coeur de ces contradictions, les instrumentalisent ou les suscitent parfois, dans ce que j'interprète- de façon certes optimistecomme une exigence historique de reconnaissance de leur autonomie.

Si l'on m'a suivie dans ces trois grandes étapes de la pensée académique sur les paysanneries en Amérique latine, on remarquera les phénomènes d'avancées -et de reculs parfois-, en tous cas de rétroalimentation entre les différentes interprétations. La seconde période -réflexion sur la nouvelle ruralité- avait représenté une avancée en termes de reconnaissance de la complexité des paysanneries et l'abandon de catégories simplificatrices, comme celle de "paysan" opposée à "agriculteur" par exemple. Il semble qu'aujourd'hui on ait tendance à retomber dans les pièges d'une certaine simplification, sous d'autres mots (indiens, noirs, acteurs ethniques), alors que les sociétés rurales continuent à évoluer sous des formes extrêmement complexes. La migration (on 
devrait dire les migrations, tant les modalités en sont diverses) et la diversification des ressources pour survivre dans des environnements en général précaires -c'est peut-être la seule constante sur ces trente années !!- suscitent des recompositions sociales et territoriales qui ont bien du mal à rentrer dans les catégories "simples" que les théories d'aujourd'hui semblent nous dessiner (par exemple "les exclus" ou "les pauvres", voire "les nouveaux pauvres").

\section{Conclusion}

On l'aura vu à l'occasion de cette esquisse ${ }^{2}$, le contexte international et ses "tendances lourdes" affectent autant le monde universitaire que politique ou économique. Faut-il s'en réjouir ou le regretter? Faut-il l'interpréter comme un conservatisme ou un "suivisme" intellectuel généralisé, ou au contraire comme la capacité des chercheurs à innover et trouver les paradigmes explicatifs les plus à même d'éclairer des situations qui se transforment tous les jours?

Ce sont les impasses théoriques d'hier qui suscitent les développements d'aujourd'hui. Il n'y a donc pas de surprise à constater l'évolution des paradigmes et aucune dénonciation à constater l'abandon de certaines catégories analytiques au profit d'autres. Au contraire, on peut y déceler des avancées conceptuelles ou théoriques sur tel ou tel point, ainsi que la traduction de transformations sociales profondes dont le monde universitaire se doit de rendre compte, que ce soit dans une optique "militante" ou plus globalement, pour une meilleure compréhension de la société dans son ensemble. La recherche permet de reconstruire le réel pour mieux le comprendre et agir sur lui, et parfois d'établir une saine distance critique entre les situations sociales et les interprétations politiques qui en sont faites à un moment donné (par exemple sur l'ethnicité). En ce sens, les évolutions soulignées ici répondent à la mission dévolue à la recherche dans les sociétés contemporaines.

Toutefois, on peut aussi voir dans l'évolution récente des théorisations un risque de fragmentation du réel - lequel est d'ailleurs lui-même questionné par certains courants comme le post-modernisme - avec le risque associé d'évacuer la dimension globale, on dirait aujourd'hui sociétale, de nos objets/sujets d'étude. Figure centrale des analyses des mondes ruraux -et nationaux- dans les années 1970, le paysan a en quelque sorte "disparu"; il s'est invisibilisé pour laisser place au producteur, au résident, au migrant, et depuis une dizaine d'années à l'indien (Bartra 1998). Le chercheur est amené à parcelliser à l'extrême ses modes de compréhension du monde s'il veut approfondir certains points théoriques, ou répondre à une demande sociale de recherche très segmentée. Prenons un exemple bassement matériel : qui aujourd'hui va financer un projet sur "les sociétés rurales" (ou paysannes), si n'apparaissent pas en bonne place les termes de genre, développement durable, pauvreté ou identité? L'approche "systémique" (années 197080) ou "globale" n'apparait plus légitime aux yeux des chercheurs ni à ceux des bailleurs de fonds. Pour les premiers, il faut "préciser" les perspectives, seule possibilité de développer concepts et méthodes sur le plan international. Pour les seconds, il faut cloisonner les problèmes

\footnotetext{
${ }^{2}$ S'agissant d'un parcours rétrospectif - et d'une vision très personnelle de celui-ci -, je n'ai pas prétention à l'exhaustivité. On m'excusera notamment de n'avoir rien dit des multiples et très bonnes études sur les transformations micro-sociales des mondes paysans (la famille, la place des femmes), sur les migrations et leurs impacts sur les structures familiales et locales de pouvoir, sur les orientations productives nouvelles ("niches" commerciales ou agriculture bio) ou sur les rapports local-global.
} 
pour permettre des actions ciblées. L'une et l'autre préoccupations semblent légitimes en soi ; elles peuvent cependant mener à une fragmentation des approches et des savoirs et à une perte de compréhension des processus globaux.

Apologie attardée des paysanneries elles-mêmes condamnées par le "développement"? Certains pourront le penser. Pourtant, c'est précisement du contraire qu'il s'agit. De prétendre qu'il est possible, à partir de l'analyse des mondes paysans et ruraux, d'éviter les catégories analytiques qui sont opératoires à court terme mais qui nous confinent dans des compartiments d'explication exclusifs, nous obligeant à laisser alors à d'autres, aux décideurs politiques de haut niveau, le soin de gérer les contradictions générées par ces juxtapositions de compréhension partielle.

FAO, Food and agriculture organisaiton

CEPAL, Comisión Económica para América Latina

OIT, Organisation Internationale du travail

PROCEDE, Programa de Certificación de Parcelas y Derechos Ejidales

Bartra, A. 1998 "Sobrevivientes historias en la frontera", Cuadernos Agrarios 16, México, pp722

Gilhodes, P. 1974 Politique et violence. La question agraire en Colombie. Librairie Armand Collin et Fondation Nationale des Sciences politiques, Paris, 537p.

Touraine, A. 1988 La parole et le sang. Politique et société en Amérique latine. Ed. Odile Jacob, Paris, 530p.

Warman, A. 1972 Los campesinos, hijos predilectos del regimen, Ed. Nuestro Tiempo, México, $138 \mathrm{p}$. 